\title{
TRABAJO SOCIAL, DIVERSIDAD SEXUAL Y ENVEJECIMIENTO. UNA INVESTIGACIÓN A TRAVÉS DE UNA EXPERIENCIA DE APRENDIZAJE-SERVICIO
}

\author{
Social work, sexual diversity and ageing. A research throught an \\ experience of service-learning
}

Josep Maria Mesquida GonZÁlez ${ }^{1}$, Violeta Quiroga RAIMÚndeZ² y ADELA BOIXADÓS PORQUET ${ }^{2}$

\section{Resumen}

Se presentan los primeros resultados de una investigación centrada en la adquisición de conocimientos acerca de la realidad de las personas mayores

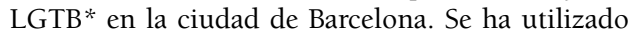
una metodología de carácter cualitativo, utilizando las técnicas de la entrevista, la entrevista grupal, el grupo de discusión y el relato de vida en una experiencia de aprendizaje-servicio en la que han participando profesores y estudiantes de Trabajo Social. En este artículo se hace una revisión de la situación del trabajo social con respecto a la diversidad sexual y se exponen elementos conceptuales que se ponen en relación con las voces de las personas que han participado en el estudio. Se constata que el grupo social del que se habla ha sido actor y testigo de excepción de una época de grandes cambios sociales habiendo realizado un proceso de tránsito desde un contexto de gran rechazo social hasta un entorno de mayor respeto y reconocimiento de derechos. También se profundiza en la homofobia y el edadismo como formas de opresión que pueden ser generadoras de déficits de salud y autonomía, así como en la inadecuación de los servicios geriátricos a las necesidades y expectativas de las personas LGTB.

Palabras clave: Diversidad sexual, LGTB, envejecimiento, trabajo social, aprendizajeservicio

* Acrónimo formado a partir de las iniciales de los grupos que componen el colectivo: lesbianas, gais, transexuales y bisexuales. Se trata de un término propuesto desde el activismo que se refiere a todas las identidades sexuales no normativas.

\section{Abstract}

We present the first results of research focused on the acquisition of knowledge about the reality of older LGBT people in the city of Barcelona. Qualitative methodology has been used: interviews, group interviews, forum groups and life stories, in a service-learning experience in which teachers and social work students participated. In this article we review the state of sexual diversity as a knowledge field of the Social Work discipline and some conceptual elements which are related to the voices of the participants in the study. It has been found that this social group has been an actor and an exceptional witness of a time of great social changes. They have lived a transition from a context of great social rejection to an environment of greater respect and recognition of rights. It also explores homophobia and ageism as forms of oppression that can cause health problems and autonomy deficits, as well as the inadequate geriatric services in relation to the needs and expectations of LGBT people.

Keywords: Sexual diversity, LGTB, ageing, social work, learning-service

1. Grupo de Investigación y Innovación en Trabajo Social (GRITS). Departamento de Trabajo Social y Servicios Sociales de la Universitat de Barcelona. Facultat de Educación. Campus de Mundet. Edifici Llevant, 3a planta. Passeig de la Vall d'Hebron, 171. 08035 Barcelona.jmesquida@ub.edu. 606452636.

2. Grupo de Investigación y Innovación en Trabajo Social (GRITS). Departamento de Trabajo Social y Servicios Sociales de la Universitat de Barcelona. Barcelona. 


\section{Introducción}

En el año 1984, el trabajador social norteamericano Raymond Berger, dirigiéndose a sus colegas de profesión, escribió un artículo titulado Realities of gay and lesbian aging. Berger perseguía despertar el interés de la profesión sobre las necesidades de las personas homosexuales mayores, considerando que padecían situaciones de vulnerabilidad en aspectos sociales, económicos y emocionales.

Desde entonces, la cuestión del envejecimiento de las personas gais, lesbianas, transexuales y bisexuales, de ahora en adelante personas LGTB, ha sido tratada por algunas y algunos científicos sociales americanos y europeos que han generado un limitado conjunto de conocimientos.

Tal y como mencionan Herdt y de Vries (2003) existirían dos perspectivas que han sido utilizadas para aproximarse al fenómeno. Un grupo importante de trabajos se basan en la idea de que la situación de las personas LGTB mayores se caracteriza por la presencia de consecuencias negativas derivadas de la discriminación padecida a lo largo de su vida. Entre ellas, una forma específica de malestar, que los angloparlantes denominan minority stress (Brooks, 1981; Meyer, 1995; Friedman, 1999) y que afectaría a grupos sociales víctimas de procesos de estigmatización.

Otro grupo importante de autores se centrarían en las fortalezas o recursos del colectivo a la hora de ajustarse al paso de los años. Las personas LGTB, en su vivencia como personas oprimidas, habrían desarrollado una forma específica de resiliencia, que recibe el nombre de mastery of crisis (Berger, 1980) o crisis competence (Friend, 1991). Esta destreza facilitaría su adaptación a nuevas formas de discriminación.

La investigación que se presenta es Personas mayores lesbianas, gais, transexuales y bisexuales en la ciudad de Barcelona que aún está en curso en el momento de la redacción de este artículo. Empieza en septiembre del 2013 y finaliza en diciembre del 2015. Participan el Grupo de Investigación e Innovación en Trabajo Social (GRITS) del Departamento de Trabajo Social y Servicios Sociales de la Universitat de Barcelona, la Fundació Enllaç y el Ayuntamiento de Barcelona.

Los objetivos de la investigación son los siguientes:

- Realizar un estudio sociodemográfico del colectivo de personas mayores LGTB en la ciudad de Barcelona.

- Analizar su situación en cuanto a necesidades sociales.

- Conocer sus redes de soporte formal e informal. 
- Conocer su grado de satisfacción y expectativas en relación a los servicios para gente mayor.

- Identificar buenas y malas prácticas de atención.

- Extraer recomendaciones que puedan servir para la mejora del plan de acción municipal LGTB de la ciudad de Barcelona.

\section{Diversidad sexual y Trabajo Social}

En nuestro entorno, el trabajo social no se ha mostrado demasiado interesado por la diversidad sexual. Pero hay algunas excepciones: destaca el libro Homosexualidad y trabajo social del trabajador social Ángel Luis Maroto (2006), quien afirma que es necesario que el trabajo social se dirija específicamente a la población homosexual considerando la diversidad sexual como un ámbito del trabajo social.

La trabajadora social norteamericana Lori Messinger (2006) sugiere que el trabajo social con el colectivo LGTB puede realizarse desde dos marcos conceptuales: la competencia cultural y la práctica del empoderamiento. Explica la competencia cultural siguiendo a Cross et al. (1989: 13) que la describen como un conjunto de conductas congruentes, actitudes, y políticas que confluyen en un sistema, agencia o entre profesionales, y admite que el sistema, la agencia, o los profesionales trabajen con eficacia en situaciones interculturales. Messinger (2006), con respecto al empoderamiento, recuerda que se trata de trabajar con las personas beneficiarias a favor del desarrollo de actitudes positivas en cuanto a su autoeficacia y su biografía personal, y a través de la sensibilización en cuanto al origen estructural de su desigualdad. Tanto la competencia cultural, como el empoderamiento, se basan en la consideración del colectivo LGTB como una minoría cultural.

La también trabajadora social norteamericana Deana Morrow (2006: 6) se pregunta si existe una cultura LGTB y recuerda a Joseph F. Healey (1997) quien defiende que la cultura engloba todos los aspectos referentes al estilo de vida asociado a un grupo de personas. Incluye el lenguaje, las creencias religiosas, las costumbres y normas sociales y los valores e ideas que la gente utiliza para organizar sus vidas e interpretar su existencia.Siguiendo la argumentación de Morrow (2006), las personas que constituyen el colectivo LGTB tienen cuatro rasgos comunes que evidencian la existencia de una cultura propia: han tenido que enfrentarse a las limitaciones derivadas de la homofobia y las interpretaciones rígidas de la expresión de género; existen instituciones dirigidas a las personas LGTB, como grupos de acción social o centros comunitarios; hay formas de expresión artística que representan particularmente a las personas 
LGTB; y finalmente, existen patrones de conducta propios del grupo que se mantienen a través de generaciones.

Por su parte, la Federación Internacional de Trabajo Social ${ }^{3}$, en su asamblea celebrada en julio del año 2014, aprobó el documento de trabajo titulado Sexual Orientation and Gender Expression ${ }^{4}$ que reconoce que las personas cuya identidad sexual, orientación sexual o expresión de género difiere de la norma son vulnerables a la opresión y a la marginalización invitando a la profesión a mejorar el nivel de respeto social hacia ellas (IFSW, 2014: 1).

El trabajo que se presenta forma parte de un conjunto de iniciativas realizadas por profesorado del Departamento de Trabajo Social y Servicios Sociales de la Universitat de Barcelona conscientes de la necesidad de incorporar contenidos relacionados con la diversidad sexual en el grado de Trabajo Social.

\section{Metodología}

El ámbito de estudio de la investigación es la diversidad sexual y el envejecimiento en la ciudad de Barcelona. Se plantea como una investigación básica, aplicada, descriptiva y explicativa, transversal y desarrollada sobre la base de una triangulación metodológica mixta (cuantitativa y cualitativa), mediante las técnicas de la encuesta en formato cuestionario, la entrevista en profundidad, la entrevista grupal, el relato de vida y el grupo de discusión. La población diana de la investigación son las personas LGTB mayores de 50 años, los profesionales de servicios geriátricos, profesionales del trabajo social, personas expertas en el tema y activistas por los derechos de las personas LGTB. Se realiza un muestreo de tipo no probabilístico intencional.

En este artículo se presentan algunos de los resultados correspondientes a la primera fase de la investigación, de tipo cualitativo. La Tabla 1 explica con detalle las técnicas utilizadas en esta fase así como las características de los informantes que han participado.

Esta primera parte se lleva a cabo en el marco del programa de aprendizaje-servicio (APS) de la Facultad de Educación de la Universitat de Barcelona. Se trata de una metodología que parte de elementos conocidos: el servicio voluntario a la comunidad y el trabajo de adquisición de conocimientos, habilidades y valores que realicen las instituciones educativas (Puig, 2012). El APS se ha desarrollado en la asignatura Investigación Aplicada al Trabajo Social ${ }^{5}$

3. En inglés: International Federation of Social Work (IFSW)

4. Disponible en http://cdn.ifsw.org/assets/ifsw_90852-5.pdf

5. En esta investigación han participado tres docentes (autores de este articulo) y 18 estudiantes: Marc Fernández, Desireé Ferrer, Sandra González, Marta RocaI, Raquel Alejandre, Xavi Berbel, Ángela Calatayud, Maria Fàbregues, Marta Fernández, Sheila Garrido, 
Tabla 1. Técnicas y características de los/as informantes

\begin{tabular}{|c|c|c|c|}
\hline Técnica & $\begin{array}{l}\text { Perfil de los/as } \\
\text { informantes }\end{array}$ & Nro. & Fecha \\
\hline $\begin{array}{l}\text { Entrevista en } \\
\text { profundidad }\end{array}$ & $\begin{array}{l}\text { Expertos del tema y } \\
\text { trabajadores sociales } \\
\text { que trabajan con } \\
\text { personas LGTB }\end{array}$ & 5 & $\begin{array}{l}\text { Octubre 2013/ } \\
\text { Noviembre } 2013\end{array}$ \\
\hline Entrevista grupal & $\begin{array}{l}\text { Personas lgtb mayores } \\
\text { de } 50 \text { años y menores } \\
\text { de } 65\end{array}$ & 4 & $18 / 11 / 2013$ \\
\hline Relato de vida & $\begin{array}{l}\text { Personas LGTB } \\
\text { mayores de } 50 \text { años }\end{array}$ & $\begin{array}{c}13 \\
\text { (5 gais, } 3 \\
\text { lesbianas y } 5 \\
\text { transexuales) }\end{array}$ & $\begin{array}{c}\text { Octubre 2013/ } \\
\text { Noviembre } 2013\end{array}$ \\
\hline \multirow{3}{*}{ Grupos de discusión } & $\begin{array}{c}\text { Activistas de } \\
\text { organizaciones LGTB }\end{array}$ & 6 & $6 / 11 / 2013$ \\
\hline & $\begin{array}{l}\text { Personas LGTB } \\
\text { mayores de } 65 \text { años }\end{array}$ & 10 & $21 / 11 / 2013$ \\
\hline & $\begin{array}{c}\text { Profesionales sector } \\
\text { geriatría }\end{array}$ & 6 & $7 / 11 / 2013$ \\
\hline
\end{tabular}

Fuente: Elaboración propia.

de $3 .^{\circ}$ curso del grado de Trabajo Social con la colaboración de la Fundació Enllaç, entidad que tiene como finalidad proteger el bienestar de las personas LGTB y de su entorno en situaciones de vulnerabilidad y dependencia, y el Ayuntamiento de Barcelona.

A lo largo del año 2014 se ha llevado a cabo la segunda fase de la investigación siguiendo una metodología cuantitativa a través de un cuestionario on line. A finales de este año, estaremos en disposición de analizar estos nuevos datos y pasaremos a elaborar un informe que combine los resultados de ambos periodos.

\section{Resultados y discusión}

A continuación se ponen en relación algunos de los resultados obtenidos con elementos conceptuales procedentes de la literatura especializada nacional e internacional. La información se presenta agrupada en torno a cuatro ejes

Laia Girós, Tania González, Judit López, Anna Manaut, Paula Martínez, Beatriz Molina, Laia Picón i Judit Román. 
temáticos que en un primer análisis emergen como cuestiones principales: la adaptación al proceso de cambio social vivido por el colectivo, la invisibilidad como resultado de una doble discriminación, la opresión vivida como posible fuente de malestar físico y emocional y la necesidad de adaptar los actuales servicios para personas mayores de forma que puedan ser más inclusivos con la realidad de las personas que integran el colectivo objeto de estudio.

\section{Transitando entre dos modelos}

En España las personas LGTB mayores han vivido trayectorias vitales que se caracterizan por su tránsito desde un modelo, el pre-gay ${ }^{6}$, en el cual predomina una homofobia severa a otro, el gay (Guasch, 1991), en el que esta homofobia dura da paso a lo que algunos autores denominan heterocentrismo u homofobia blanda (Guasch, 1991; Arnalte, 2003; Calvo, 2003; Ugarte, 2011). También han pasado de ser consideradas como pecadoras, delincuentes y enfermas (Benito y Villagrasa, 2009) a ser tratadas con igualdad jurídica en muchos aspectos.

Óscar Guasch (1991) explica la transformación vivida por los hombres gais describiendo dos modelos. La identidad homosexual vigente durante la dictadura y primeros años de la transición recibiría el nombre de modelo Pre-gay. A lo largo de este periodo, los hombres y mujeres homosexuales y transexuales padecen una marginación específica (Ugarte, 2008) que tiene las siguientes características: la discriminación legal se superpone a la marginación social; las personas homosexuales no son delincuentes políticos, sino sociales, siendo portadores de un estigma que no tienen los otros; los homosexuales no constituyen grupos específicos en ningún colectivo organizado; y finalmente, la soledad de los homosexuales es superior a la de cualquier otro grupo marginado porque no cuenta con el soporte de ninguna institución.

Tal y como muestra Kerman Calvo (2013), a partir del inicio de la transición, la agenda política española empieza a asumir la situación de las personas homosexuales como asunto de interés en paralelo a un proceso general de mayor aceptación social. Las personas homosexuales en el periodo Gay (Guasch, 1991) o lgbt (Ugarte, 2011) comienzan a hacer un uso generalizado de instituciones definiendo unos límites claros de los espacios de relación.

Los mayores LGTB relatan trayectorias de vida que se mueven desde un modelo al otro. Se describen infancias y juventudes inmersas en un contexto

6. Se utiliza el término anglosajón gay, en lugar del castellano gai, cuando se hace referencia al modelo nacido en Estados Unidos. Se pretende evidenciar de este modo su carácter importado. 
con roles de género sexistas muy rígidos: «Tú debías hacer lo que tenías que hacer, lo que se supone que te tocaba» (Gai. 61 a. RV: 30/11/2013) ${ }^{7}$. En algunos casos se menciona la ausencia de referentes homosexuales: «No sabía lo que era un homosexual, yo» (Gai. 82 a. RV: 30/10/2013), «Le di nombre a los 16 años, cuando estaba estudiando 5. ${ }^{\circ}$ de bachiller» (Lesbiana. 59 a. RV: 30/10/2013).

Se refieren relaciones de familia en las que la sexualidad de las personas entrevistadas es un tema del cual no se habla: "Intenté decírselo a mi madre: "Bueno mamá, es que tú ya sabes que"..... ella dijo "Sí, sí... no es necesario que me expliques nada"» (Lesbiana. 67 a. RV: 31/11/2013).

Se explican situaciones de total incomprensión por parte de las personas que configuran su entorno relacional: "Y esta chica, que era compañera de clase, a quien le dije un día te quiero porque necesitaba soltarlo, pero no supe decirle de qué manera la quería, iy ella tampoco lo entendió! Se echó a reír, no entendió nada...» (Lesbiana. 59 a. RV: 30/10/2013).

Los participantes en el estudio han sido testigos y actores del cambio de modelo. "Habia mucha ilusión, la gente tenía ganas de libertad, empezaron a aparecer locales por todas partes» (Lesbiana. 59 a. RV: 30/10/2013). En algunos casos, se narran las migraciones producidas por la búsqueda de un entorno más respetuoso en Barcelona. En donde las transformaciones sociales se suceden con rapidez siendo fácil mantener el anonimato o moverse por circuitos específicos: «Fue una inmersión total. Llegué a Barcelona (...). Tenía claro qué tipo de vida quería. Entonces fui a l'Institut Lambda. Conocí a todo el mundo y empecé a salir. Hacía de todo» (Gai. 61 a. RV: 30/11/2013). La evolución política también favorece la articulación del activismo lésbico en torno al movimiento feminista: «En aquel momento comienza la segunda ola del feminismo. Nos identificamos como feministas y entonces vemos que en movimiento feminista coincidimos con otras mujeres lesbianas» (Lesbiana. 67 a. RV: 31/11/2013).

Se reconocen las mejoras producidas a nivel social y legal y la forma en que éstas han impactado positivamente en su bienestar. "Ahora estoy viviendo la juventud que no viví, como en la gran obra $<<A$ la recherche du temps perdu>>» (Gai. 87 a. RV: 30/11/2013). "No hace ni un año que estoy casado. Es mi pareja desde hace ocho años y me ha cuidado siempre. Es una persona excelente, me da todo el soporte que necesito» (Gai. 69 a. RV: 5/11/2013). Pero también manifiestan temor a que se produzcan retrocesos en cuanto a libertad y respeto hacia el colectivo: «Las cosas no las tenemos ganadas para siempre» (Lesbiana. 67 a. RV: $31 / 11 / 2013$ ).

7. Para referenciar las citas textuales se ha hecho uso de la siguiente codificación: entrevista en profundidad (E), entrevista grupal (EG), grupo de discusión (GD) y relatos de vida (RV). 
Homofobia y edadismo: doble invisibilidad

Las personas mayores LGTB padecen dos formas de discriminación: la homofobia ${ }^{8}$ y el edadismo. La homofobia, siguiendo a Daniel Borrillo (2000) sería la actitud hostil respecto a las personas homosexuales. No sólo haría referencia a acciones claramente violentas, sino también a situaciones mucho más cotidianas y, por tanto, más difíciles de detectar, incluiría actitudes afectivas y cognitivas hacia la homosexualidad.

Por otro lado, el edadismo sería una forma de discriminación basada en la edad (Sagrera, 1992; Butler, 1989). La separación de los mayores de los espacios de producción y de ocio puede considerarse prácticas edadistas.

Hay dos estereotipos que se combinan. El primero presenta a las personas homosexuales como sujetos centrados en la satisfacción de sus deseos sexuales. El estilo de vida homosexual se caracterizaría por el disfrute constante de aventuras sin compromiso en entornos destinados al encuentro sexual y otras manifestaciones fundamentalmente hedonistas. Se trata de una creencia homófoba porque reduce a las personas homosexuales a seres sin más dimensión que la sexual.

El segundo estereotipo es de origen edadista y apunta hacia la consideración de los mayores como personas sin vida sexual. Serían personas sin posibilidades en el mercado sexual.

Si las personas LGTB son hombres y mujeres casi absolutamente centrados en la satisfacción de sus deseos eróticos, y las personas mayores no tienen sexo, se puede inferir que las personas homosexuales mayores no existen. Esta conclusión podría ayudar a explicar el hecho de que la población mayor LGTB sea un grupo social invisible.

Una educadora en una residencia de gente mayor expresaba: «Yo creo que es necesario (que) se visualicen. Es una de las cosas que necesitan por encima de todo. La invisibilidad que padecen. Sobre todo en residencias y centros sociosanitarios» (GD: 7/11/2013)

También se ha puesto en evidencia la marginación que las personas LGTB mayores padecen por parte del propio colectivo. Tal y como dice un trabajador social y activista LGTB, «En la comunidad LGTB los mayores no tienen

8. Nos adherimos a la propuesta de Borrillo (2000: 16) y hacemos uso del término homofobia en un sentido amplio, refiriéndose a cualquier forma de violencia, incluida la simbólica (Bordieu, 2000) por motivos de orientación sexual o identidad de género. Evitamos el uso de conceptos como bifobia y transfobia entendiendo que la violencia homófoba afecta no sólo a las personas homosexuales, sino a todas aquellas que no se ajustan al orden clásico de los géneros. 
lugar social. Sólo se genera un espacio en la comunidad LGTB que es para gente joven» (E: 30/10/2013).

\section{Malestar emocional}

La investigación en salud de la población LGTB es escasa (Boehmer, 2002; Addis, Myfanwy, Greene, Mac Bride-Stewart, y Shepherd, 2009; FredriksenGoldsen et al. 2013). Y podemos calificarla de casi inexistente si nos referimos específicamente a personas transexuales y bisexuales (Warner, Mc Keown, Griffin, Johnson, Ramsay, Cort, 2004). Un análisis de los trabajos realizados durante los 5 años anteriores al de la investigación de referencia en las 10 revistas de enfermería con mayor factor de impacto muestra que sólo el 0,16\% de los artículos concierne al colectivo LGTB (Gabrielson, 2011). La orientación sexual no ha sido un dato considerado relevante para ser recogido en los paquetes básicos de datos incorporados por las administraciones sanitarias. Por otro lado, el temor a la discriminación hace que esta cuestión no se manifieste en las relaciones de la persona con el sistema sanitario (Addis et al., 2009).

Diversos estudios han intentado evaluar las necesidades de las personas mayores LGTB, a través de un análisis sistemático de la literatura (Addis et al., 2009; Brotman, Ryan, y Cormier, 2003; Orel, 2004) y en ellos aparecen algunos problemas de salud de forma reiterada. Así, se da en el estudio de Fredriksen-Goldsen et al. (2013), cuyos resultados muestran unos niveles de salud general bajos $(24,13 \%)$, una alta incidencia de discapacidad $(45,49 \%)$ y de depresión (29\%). También en esta línea, Lick, Durso y Johnson (2013) indican que existe una relación directa entre las consecuencias de esta discriminación, victimización, internalización del estigma, discriminación laboral y la salud mental que conlleva una prevalencia mayor de trastornos de la ansiedad, de depresión y de consumo de tóxicos. En un estudio realizado con la participación de 1285 personas LGTB, el 43\% manifestaba tener alguna alteración psiquiátrica y el 31\% había realizado alguna tentativa de suicidio (Warner, et al., 2004).

A nivel local, la Encuesta de Salud de Barcelona 2011 (Pérez et al., 2014), incluye por primera vez preguntas en relación con la orientación sexual, lo que permite obtener datos sobre el estado de salud de 131 personas (4,04\% de la muestra) que manifiestan haber mantenido relaciones sexuales con personas del mismo sexo. Los resultados también mostraron las mismas conclusiones expuestas anteriormente: peor salud general, mayor índice de ansiedad y depresión, y mayor consumo de sustancias tóxicas.

Algunas de las personas LGTB entrevistadas refieren malestar e infelicidad. "Estoy hecha mierda. Es una situación horrorosa. Este verano ha sido el 
peor. En agosto deseaba no despertarme. Porque a ver ¿qué hago? Dónde está mi salida que no la encuentro» (M. transexual. 63 a. RV: 25/11/2013). "Yo no sé si es tristeza o lo que es, pero me encuentro con mucha angustia» (Gai. 80 a. GD: 21/11/2013). También otra persona relaciona su malestar con el hecho de sentirse solo. «Me levanto desde hace muchos años muy malo... hace ya 20 años que estoy siempre solo» (Gai. 68 a. GD: 21/11/2013).

La soledad ha aparecido como una fuente frecuente de malestar: «La soledad afectiva para mí es lo peor. Estoy muy jodido. Llega un momento con una edad que no puedo hacer amistades nuevas, porque una amistad necesita su tiempo» (Lesbiana. 69 a. GD: 21/11/2013). Otra persona entrevistada decía «Yo pienso que la soledad es un virus. Por eso, si pienso en un futuro me entristezco» (M. Transexual. 61 a. RV: 18/11/2013).

Otro elemento a considerar es el envejecimiento de las personas LGTB en tratamiento por la infección por el VIH. En España, la organización BCN Checkpoint declaraba en el mes de julio del año 2014 que la incidencia del virus aumentaba un $7 \%$ anualmente entre la población homosexual representando los varones gais un $60 \%$ de los nuevos infectados ${ }^{9}$. La infección por VIH se asocia con distintos problemas de salud relacionados con la infección o con su tratamiento que requieren una atención especial. La condición de seropositivo puede añadir un elemento más de discriminación hacia este grupo de personas. Otro elemento a considerar es la debilidad de la red social de aquellas personas que la vieron diezmada en los años 80 y 90 del siglo pasado en los momentos más duros de la epidemia (Jablonski, Vance, y Beattie, 2013).

Tal y como afirma uno de los trabajadores sociales participantes, "Con el tema del VIH ha habido mucha gente que ha perdido a todo su entorno. Esto en el mundo de las lesbianas no es así, pero en el mundo de hombres gais y de personas transexuales, el VIH ha sido una escabechina» (E: 10/20/2013).

Finalmente, hay que hacer especial mención a la situación de las mujeres transexuales, que a menudo presentan una muy baja calidad de vida relacionada con las carencias económicas. Así, una mujer transexual activista comentaba «Desde la perspectiva transexual, hay una necesidad específica, porque la mayoría de ellas nunca han trabajado o no les han dejado trabajar: no han cotizado... Llegan a los 65-70 años, no tienen ayudas y se encuentran en la calle» (M. transexual. 54 a. GD: 06/11/2013).

9. Según noticia aparecida en el periódico El País el día 3 de julio del año 2014: http://ccaa. elpais.com/ccaa/2014/07/02/catalunya/1404330160_179762.html 
Acerca de servicios poco adecuados

La pérdida de la autonomía muchas veces conlleva el uso de servicios específicos que proporcionan diferentes niveles de apoyo, tanto a las personas afectadas como a sus familias. La utilización de estos recursos implica la entrada de profesionales e instituciones en aspectos y espacios personales e íntimos. Además, muchas veces se obliga a las personas atendidas a respetar una serie de requisitos que pueden entrar en colisión con sus preferencias, con el ejercicio de su libertad (Bazo, 1991).

Los servicios para la dependencia constituyen uno de los grandes temas presentes en la literatura sobre las necesidades de las personas LGTB. Hay diferentes cuestiones que se incluyen en esta categoría: la no adecuación de los mismos a las necesidades de este colectivo; la forma en que se pueden adaptar los recursos existentes, orientados a la población general; y la necesidad y creación de servicios específicos.

Los servicios sanitarios y sociales diseñados para atender a las personas mayores son considerados como poco satisfactorios. Se reclama que reconozcan la diversidad del grupo y el contexto social en el que han de desarrollarse (Beeler, Rawls, Herdt y Cohler 1999; Hugues, 2008). También se pone de manifiesto que la expectativa de discriminación en los servicios sociales y de salud puede haber provocado un uso inferior de los mismos, originando situaciones de desatención de personas dependientes o sobrecarga por parte de sus cuidadores (Brotman, et al., 2003). Según Cheese (2010) la invisibilidad de las personas LGTB en la sociedad en general, así como la discriminación por edad entre sus iguales, conlleva que las respuestas que proporcionan los modelos actuales de servicio y atención estén muy lejos de lo que el colectivo necesita.

La gran mayoría de los profesionales opina que es preferible tratar de adaptar los servicios existentes que crear otros específicamente dirigidos al colectivo. Pero hay quien considera que estos últimos son necesarios actualmente, cuando todavía es preciso mejorar el nivel de respeto hacia la diversidad sexual para garantizar que sean espacios seguros. Así, otro de los trabajadores sociales participantes indica que "Quizá vale la pena crear servicios específicos si ahora son necesarios» (E: 20/10/2013). Y una mujer lesbiana participante manifiesta que "Los servicios no están preparados o todavía la gente no tiene el chip de aceptar que existe esta diversidad sexual» (Lesbiana. 50 a. EG: 18/11/2013). Una profesional del ámbito geriátrico que participo en un grupo de discusión indica "La mayoría de gente mayor que está en las residencias son de una generación que culturalmente han vivido un rechazo hacia esto y les cuesta muchísimo aceptarlo.» (GD: 07/11/13). Otra profesional del trabajo 
social expresa que «La gente mayor prefiere servicios en dónde sean iguales que ellos, para que se les respete, para que puedan sentirse acogidos y no tener que ir escondiendo su identidad sexual» (E: 31/11/2013).

En el grupo de discusión de personas mayores LGTB se reclama formación dirigida a sensibilizar en torno al respeto hacia la diversidad sexual. Un activista de la entidad Asociación catalana para la integración de homosexuales, bisexuales y transexuales inmigrantes (ACATHI), que se dedica a las personas LGTB migrantes, indica que "El aislamiento que se siente es mucho más exagerado, a la vez que se presentan los servicios, y que no tienen respuestas a sus necesidades, y por consecuencia se sienten mucho más fuera del sistema» (GD: 6/11/2013).

Refiriéndose a las personas transexuales, autores como Higgins, et al. (2011), Israel y Tarver (1997) y Meyer (2001) expresan que este grupo puede tener problemas particulares con respecto a los servicios a medida que envejecen. Se destaca la importancia de educar a los profesionales de la asistencia sanitaria y social sobre las necesidades de las personas mayores transexuales, incluyendo salud física y emocional (Witten, 2002).

Las personas transexuales preguntadas sobre la necesidad de recursos específicos expresan que les gustaría que éstos fueran destinados específicamente a personas LGTB. Emerge la idea de que debería haber viviendas compartidas como opción preferible a la de una residencia. Daniela manifiesta «Me planteé mí futuro en una cooperativa de personas mayores en la cual nos podemos alquilar un piso y tiene unos servicios de miedo, pero yo no tengo un fondo que me permite acceder, dependo de los Servicios Públicos» (M. transexual. 61 a. RV: 18/11/2013).

El sociólogo Jordi Monferrer (2010), refiriéndose a los temas que aparecen como nuevos marcos de movilización de las entidades LGTB, señala la atención a las personas mayores del colectivo como uno de ellos.

Se desea que el propio colectivo genere recursos reconociendo el trabajo realizado por organizaciones como la Fundació Enllaç, el Casal Lambda, el Grup de Amics Gais, Gais Positius, La Nostra Illa y Ca la Dona. Se trata, en su mayoría, de proyectos que nacen en el seno de organizaciones LGTB o feministas que habitualmente han sido espacios reivindicativos y asociativos. También se mencionan iniciativas como la Fundació Enllaç, creada específicamente para dar respuesta a las personas mayores $\mathrm{LGTB}^{10}$ en Catalunya.

10. Otro ejemplo de organización LGTB que se dedica a los mayores es la Fundación 26 de Diciembre en Madridhttp://www.fundacion26d.org/. Existen iniciativas parecidas en otros países, como SAGE, en Estados Unidos, http://sageusa.org/about/. 


\section{Conclusiones y recomendaciones}

Las personas LGTB mayores son un grupo social poseedor de una vivencia muy particular, porque han vivido un período de gran cambio social que les ha permitido pasar del rechazo y el silencio a mayores niveles de respeto y presencia pública, porque deben afrontar una doble discriminación basada en la combinación de la homofobia y el edadismo, porque han padecido expresiones de profundo rechazo que puede haber impactado en sus vidas en forma de malestar físico y emocional y, finalmente, porque miran hacia los actuales servicios de atención a las personas mayores con miedo a perder la libertad ganada.

A pesar de que el trabajo social apenas se ha ocupado de la diversidad sexual como ámbito de intervención, se muestran evidencias que sugieren la necesidad de poner en marcha acciones dirigidas a paliar aquellas situaciones que parecen afectar al colectivo, como mayores niveles de malestar emocional y soledad con relación a sus iguales heterosexuales.

Igualmente parece aconsejable proporcionar espacios de relación intergeneracional entre personas LGTB para tratar de mejorar la consideración de los más jóvenes en relación a sus mayores.

También se evidencia la necesidad de repensar los actuales servicios de atención a mayores haciendo de ellos entornos que sean percibidos como espacios seguros por la población homosexual y transexual, y se invita a reflexionar en torno a la posibilidad de generar servicios específicos.

El estudio, una vez haya finalizado, debe arrojar mayor luz acerca de la relación entre la opresión vivida y la aparición de determinados trastornos. Tanto la literatura consultada como el propio testimonio de las personas participantes en esta primera fase, sugieren que los actuales niveles de diferentes tipos de malestares, que son mayores que los que padece la mayoría no LGTB, pueden tener su origen en la experiencia de estas personas como minoría discriminada.

Finalmente, queremos poner en valor la experiencia del APS como oportunidad, no sólo referida a la adquisición de conocimientos y habilidades, sino también a la formación en aspectos éticos, como el respeto a la diversidad en general, la necesidad de mirar hacia la realidad social teniendo en cuenta la mirada del otro, así como el compromiso de la profesión con la divulgación de nuevos problemas sociales, de cuestiones de las que todavía se habla poco y que emergen como posibles nuevas preocupaciones. 


\section{Bibliografía}

Addis, S., Myfanwy, D., Greene, G., MacBride-Stewart, S., y Shepherd, M. (2009). The health, social care and housing needs of lesbian, gay, bisexual and transgender older people: a review of the literature. Health and Social Care in the Community, 17(6), 647-658.

ARNalte, A. (2003). Redada de violetas: la represión de los homosexuales durante el franquismo. Madrid: La esfera de los libros.

BAzO, M. T. (1991). Institucionalización de personas ancianas: un reto sociológico. Reis, 149-164.

BeEler, J. A., RaWls, T. W., Herdt, G., \& COHLER, B. J. (1999). The needs of older lesbians and gay men in Chicago. Journal of Gay $\mathcal{E}$ Lesbian Social Services, $9(1), 31-49$.

Benito, J. y Villagrasa, C. (2009). Los otros represaliados y discriminados del franquismo, desde la memoria histórica. Barcelona: Edicions Bellaterra

BERGER, R. M. (1980). Psychological adaptation of the older homosexual male. Journal of Homosexuality, 5(3), 161-176.

- (1984). Realities of gay and lesbian aging. Social Work, 29 (1), 57-62.

BoeHmer, U. (2002). Twenty Years of Public Health Research: Inclusion of Lesbian, Gay, Bisexual, and Transgender Populations. American Journal of Public Health, 92(7), 1125-1130.

BOURDieu, P. (2000). La dominación masculina. Barcelona: Anagrama.

Borrillo, D. (2000). Homofobia. Barcelona: Edicions Bellaterra.

Brotman, S., RyAn, B., y CORMiER, R. (2003). The health and social service needs of gay and lesbian elders and their families in Canada. The Gerontologist, 43(2), 192-202.

Brooks, Virginia R. (1981). Minority Stress and Lesbian Women. Lexington, MA: Lexington Books, D.C. Heath and Co.

Butler, R. N. (1989). Dispelling ageism: The cross-cutting intervention. The Annals of the American Academy of Political and Social Science, 138-147.

Calvo, K. (2003). Actitudes sociales y homosexualidad en España, en O. Guasch i O. Viñuales, Sexualidades. Diversidad y control social. Barcelona: Bellaterra.

- (2013). Antes de que fuéramos familias: mordazas, homosexualidad y debates parlamentarios en España (1978-1995). En Minorías sexuales en España (1970-1995): textos y representaciones (pp. 27-45). Icaria.

Cross, T. L., Bazron, B. J., Dennis, K. W., \& IsaAcs, M. R. (1989). Towards a Culturally Competent System of Care: A Monograph on Effective Services for Minority Children Who Are Severely Emotionally Disturbed. Washington, DC: Georgetown University, Child Development Center, Child and Adolescent Service System Program, Technical Assistance Center. 
CHEESE, B. (2010). Improving the Lives of Lgbt Older Adults. en https://www.sageusa.org/files/Improving\%20the\%20Lives\%20of\%20LGBT\%20Older\%20 Adults\%20-\%20full\%20report.pdf (1/10/2014).

Fredriksen-Goldsen, K., Emlet, C.A., Kim, H.-J., Muraco. A., Erosheva, E.A., GOLDSEN, J., et al. (2013). The physical and mental health of lesbian, gay male, and bisexual (LGB) older adults: the role of key health indicators and risk and protective factors. The Gerontologist 53(4), 664-675.

FRIEND, R. A. (1991). Older lesbian and gay people: A theory of successful aging. Journal of Homosexuality, 20, 99-118.

FrIEDMAN, R. C. (1999). Homosexuality, psychopathology, and suicidality. Archives of General Psychiatry, 56(10), 887-888.

GABrielson, M.L. (2011). I Will Not Be Discriminated Against» Older Lesbians Creating New. Advances in Nursing Science, 34(4), 357-373.

GUASCH, O. (1991). La sociedad rosa. Barcelona: Anagrama.

HeAley, J. F. (1997). Race, ethnicity, and gender in the United States: Inequality, group conflict, and power. Thousand Oaks, CA: Pine Forge Press.

HeRDT, G. H. y DE VRIES B. (2003). Gay and lesbian aging: Research and future directions. Springer Publishing Company.

Higgins, A., Sharek, D., McCann, E., Sheeran, F., Glacken, M., Breen, M., y MC CARron, M. (2011). Visible lives: Identifying the experiences and needs of older lesbian, gay bisexual and transgender people in Ireland. Dublin: Gay and Lesbian Equality Network.

HuGHES, M. (2008). Imagined futures and communities: Older lesbian and gay people's narratives on health and aged care. Journal of Gay E Lesbian Social Services, 20(1-2), 167-186.

IFSW (2014). Sexual Orientation and Gender Expression. Draft 5/29/14. Disponible en http://cdn.ifsw.org/assets/ifsw_90852-5.pdf (10/10/2014).

ISRAEL, G. E., y TARVER, D. E. II. (1997). Transgender care: recommended guidelines, practical information, and personal accounts. Temple University Press, Philadelphia.

Jablonski, R., VAnCE, D.E., y BEAtTIE, E. (2013).The invisible elderly. Lesbian, gay, bisexual, and transgender older adults. Journal of Gerontological Nursing, 39(11), 46-52.

Lick, D.J., Durso, L.E., y Johnson, K.L. (2013). Minority Stress and Physical Health Among Sexual Minorities. Perspectives on Psychological Science, 8(5), 521-548.

MAROto SÁEZ, A. L. (2006). Homosexualidad y trabajo social: Herramientas para la reflexión e intervención profesional. Madrid: Consejo General Diplomados en Trabajo Social y Asistentes Sociales. 
Messinger, L. (2006). Toward affirmative practice. Sexual orientation and gender expression in social work practice: Working with gay, lesbian, bisexual, and transgender people, 460-470.

MeYer, I. H. (1995). Minority stress and mental health in gay men. Journal of health and social behavior, 38-56.

- (2001). Why lesbian, gay, bisexual, and transgender public health?. American Journal of Public Health, 91(6), 856.

MONFERRER J. (2010). Identidad y cambio social: transformaciones promovidas por el movimiento gay/lesbiano en España. Barcelona-Madrid: Egales Editorial.

Morrow, D. F. (2006). Sexual orientation and gender identity expression. Sexual orientation $\&$ gender expression in social work practice: Working with gay, lesbian, bisexual, \& transgender people, 3-17. New York: Columbia University Press.

Orel, N.A. (2004). Gay, lesbian, and bisexual elders: expressed needs and concerns across focus groups. Journal of Gerontological Social Work, 43(2/3), 57-77.

Pérez, G., Martí-Pastor, M., Gotsens, M., Bartoll, X., Diez, E., \& Borrell, C. (2014). Salud y conductas relacionadas con la salud según la atracción y la conducta sexual. Gaceta Sanitaria.

PUIG J. M. (2012). Compromís cívic i aprenentatge a la universitat (Vol. 185). Barcelona: Grao.

QuAM, J. K., \& WHITFORD, G. S. (1992). Adaptation and age-related expectations of older gay and lesbian adults. The Gerontologist, 32(3), 367-374.

SAGRERA, M. (1992). El edadismo: contra «jóvenes» y «viejos»: la discriminación universal. Madrid: Editorial Fundamentos.

UgARTE PÉREZ, J. (2008). Una discriminación universal: la homosexualidad bajo el franquismo y la Transición. Barcelona-Madrid: Egales Editorial.

- (2011). Homoerotismo, identidad y resistencia. Madrid-Barcelona: Egales.

Warner, J., Mc Keown, É., Griffin, M., Johnson, K., Ramsay, A., Cort, C., et al. (2004). Rates and predictors of mental illness in gay men, lesbians and bisexual men and women. British Journal of Psychiatry, 185, 479-485.

WitTen, T. M. (2002). Midlife issues of aging in the LGBT community: A roundtable discussion. Outword, 20, 98-97. 